\title{
Neutrino Mass Hierarchies in a Mass Matrix Form Versus its Inverse Form
}

\author{
Yoshio Koide \\ IHERP, Osaka University, 1-16 Machikaneyama, Toyonaka, Osaka 560-0043, Japan \\ E-mail address: koide@het.phys.sci.osaka-u.ac.jp
}

\begin{abstract}
A neutrino mass matrix model $M_{\nu}$ with $M_{\nu}^{T}=M_{\nu}$ and a model with its inverse matrix form $\widetilde{M}_{\nu}=m_{0}^{2}\left(M_{\nu}^{*}\right)^{-1}$ can be diagonalized by the same mixing matrix $U_{\nu}$. It is investigated whether a scenario which provides a matrix model $M_{\nu}$ with normal mass hierarchy can also give a model with an inverted mass hierarchy by considering a model with an inverse form of $M_{\nu}$.
\end{abstract}

It is very interesting problem in the current neutrino physics whether neutrino mass hierarchy is normal or inverted. If the observed neutrinos are of Majorana type, models with an inverted hierarchy will be testable by observing the effective neutrino mass $\left\langle m_{e e}\right\rangle$ in the near future neutrinoless double beta decay experiments because we expect $\left\langle m_{e e}\right\rangle \sim \sqrt{\Delta m_{\text {atm }}^{2}} \simeq 0.05$ $\mathrm{eV}$. On the other hand, if we cannot observe a non-vanishing value of $\left\langle m_{e e}\right\rangle$ up to $\sim 0.01 \mathrm{eV}$, as far as the hierarchy is concerned, its test will be hopeless in the near future experiments. For the prediction whether the neutrino mass hierarchy is normal or inverted, current models are half and half. However, in this paper, we notice that, in a model with a mass matrix form $M_{\nu}\left(M_{\nu}^{T}=M_{\nu}\right)$ which can provide reasonable masses $m_{\nu i}(i=1,2,3)$ and mixing $U_{\nu}$, a mass matrix with an inverse form $\widetilde{M}_{\nu}=m_{0}^{2}\left(M_{\nu}^{*}\right)^{-1}$ can give the same mixing $U_{\nu}$ as in the original model $M_{\nu}$. This means a possibility that a model with a normal mass hierarchy can also give an inverted hierarchy. Then, most current models will be able to predict an inverted hierarchy, i.e. most models have a possibility $\left\langle m_{e e}\right\rangle \sim 0.05 \mathrm{eV}$. This will encourage experimental physicists who have a plan to measure $\left\langle m_{e e}\right\rangle$ with the order of $0.05 \mathrm{eV}$.

Such a scenario with "dual" hierarchy is the following case: Let us consider an effective neutrino mass matrix $M_{\nu}$ on a flavor basis on which the charged lepton mass matrix $M_{e}$ is diagonal (or nearly diagonal). The mass matrix form $M_{\nu}$ is invariant under a flavor basis transformation $T$, i.e.

$$
T^{T} M_{\nu} T=M_{\nu}
$$

We may consider such operators $T$ more than two. In other words, we consider that the mass matrix $M_{\nu}$ has been derived from the constraints (1). Since we know a no-go theory [1] for flavor symmetries, we consider that the mass matrix $M_{\nu}$ is an effective type, e.g. the form $M_{\nu}$ is described in terms of Higgs scalars more than two, or it is of a Froggatt-Nielsen type [2]. (Therefore, the matrices $T$ can operate only to neutrino sector independently of the charged lepton sector.) We also define a neutrino mass matrix $\widetilde{M}_{\nu}$ as

$$
\widetilde{M}_{\nu}=m_{0}^{2}\left(M_{\nu}^{*}\right)^{-1}+m_{0} \xi_{0} \mathbf{1}
$$


From the inverse of Eq.(1), we obtain

$$
T^{T}\left(M_{\nu}^{*}\right)^{-1} T=\left(M_{\nu}^{*}\right)^{-1}
$$

so that

$$
T^{T} \widetilde{M}_{\nu} T=m_{0}^{2}\left(M_{\nu}^{*}\right)^{-1}+m_{0} \xi_{0} T^{T} T .
$$

Therefore, for a case that the matrices $T$ are orthogonal (e.g. an exchange operator between $\nu_{\mu}$ and $\nu_{\tau}$ in the 2-3 symmetry [3], and so on), we obtain

$$
T^{T} \widetilde{M}_{\nu} T=\widetilde{M}_{\nu}
$$

i.e. the mass matrix form $\widetilde{M}_{\nu}$ is also allowed under the same symmetry. (Note that for operators $T$ with $T^{T} T \neq \mathbf{1}$ the matrix $\widetilde{M}_{\nu}$ is not invariant under the symmetry. For such a general case with $T^{T} T \neq 1$, the invariance (5) holds only when $\xi_{0}=0$. On such a case, we will comment later.)

On the other hand, the neutrino mass matrix $M_{\nu}$ is diagonalized as

$$
U_{\nu}^{T} M_{\nu} U_{\nu}=D_{\nu} \equiv \operatorname{diag}\left(m_{1}, m_{2}, m_{3}\right) .
$$

Therefore, the matrix $\widetilde{M}_{\nu}$ is also diagonalized as

$$
U_{\nu}^{T} \widetilde{M}_{\nu} U_{\nu}=m_{0}^{2}\left(D_{\nu}^{*}\right)^{-1}+m_{0} \xi_{0} U_{\nu}^{T} U_{\nu}=\operatorname{diag}\left(\widetilde{m}_{1}, \widetilde{m}_{2}, \widetilde{m}_{3}\right),
$$

where we have assumed that $M_{\nu}$ is real matrix, so that $U_{\nu}^{T} U_{\nu}=\mathbf{1}$. Therefore, we can obtain the same neutrino mixing matrix $U_{\nu}$ for $\widetilde{M}_{\nu}$, too. The mass eigenvalues $\widetilde{m}_{i}$ of $\widetilde{M}_{\nu}$ are given by

$$
\widetilde{m}_{i}=m_{0}\left(\frac{m_{0}}{m_{i}}+\xi_{0}\right)
$$

When we define the ratio $R$

$$
R \equiv \frac{m_{2}^{2}-m_{1}^{2}}{m_{3}^{2}-m_{2}^{2}}
$$

correspondingly to the observed ratio $R_{o b s}=\Delta m_{\text {solar }}^{2} / \Delta m_{\text {atm }}^{2}$, we obtain

$$
\widetilde{R} \equiv \frac{\widetilde{m}_{2}^{2}-\widetilde{m}_{1}^{2}}{\widetilde{m}_{3}^{2}-\widetilde{m}_{2}^{2}}=\left(\frac{m_{3}}{m_{1}}\right)^{2} \frac{1+2 \xi_{0} m_{2} m_{1} /\left(m_{2}+m_{1}\right) m_{0}}{1+2 \xi_{0} m_{3} m_{2} /\left(m_{3}+m_{2}\right) m_{0}} R .
$$

Therefore, we can, in general, fit the value of $\widetilde{R}$ to the observed value by adjusting the free parameter $\xi_{0} / m_{0}$ suitably. In fact, we can obtain $\widetilde{R}=\mp R$ by choosing the parameter $\xi_{0} / m_{0}$ as

$$
\frac{\xi_{0}}{m_{0}}=\frac{\left(m_{3}^{2} \pm m_{1}^{2}\right)\left(m_{3}+m_{2}\right)\left(m_{2}+m_{1}\right)}{2 m_{3} m_{2} m_{1}\left[m_{3}\left(m_{3}+m_{2}\right) \pm m_{1}\left(m_{2}+m_{1}\right)\right]}
$$


We have an interest in whether a model $\widetilde{M}_{\nu}$ gives an inverted mass hierarchy (IH) or not when the original model $M_{\nu}$ gives a normal mass hierarchy $(\mathrm{NH})$. For convenience, we define that $m_{2}^{2}>m_{1}^{2}$ (so that the model gives $\tan ^{2} \theta_{\text {solar }} \sim 0.5$ ), so that a case with NH gives $R>0$. Since the mixing matrix $U_{\nu}$ is identical both for $M_{\nu}$ and $\widetilde{M}_{\nu}$, the masses $\widetilde{m}_{i}$ have also to satisfy the relation $\widetilde{m}_{2}^{2}>\widetilde{m}_{1}^{2}$ because $\widetilde{M}_{\nu}$ will also give $\tan ^{2} \theta_{12} \sim 0.5$. Therefore, $\widetilde{R}$ takes $\widetilde{R}>0$ or $\widetilde{R}<0$ according as $\mathrm{NH}$ or IH in $\widetilde{M}_{\nu}$ as well as those in $M_{\nu}$. In order to see whether the model $\widetilde{M}_{\nu}$ gives $\mathrm{NH}$ or $\mathrm{IH}$, we calculate $\widetilde{x} \equiv \widetilde{m}_{2} / \widetilde{m}_{3}$ correspondingly to $x \equiv m_{2} / m_{3}$. If we see $\widetilde{x}^{2} \ll 1$ $\left(\widetilde{x}^{2} \gg 1\right)$ for $x^{2} \ll 1$, the case describes a model with NH (IH). We can describe the value $\widetilde{x}$ as a function of $x$ by using the constraint (11):

$$
\widetilde{x}=-\frac{\frac{m_{2}-m_{1}}{m_{2}+m_{1}}+\frac{m_{2}-m_{1}}{m_{3}}+\frac{m_{2} m_{1}}{m_{3}^{2}}}{1+\frac{m_{2}^{2}+m_{1}^{2}}{\left(m_{2}+m_{1}\right) m_{3}}-\frac{m_{2} m_{1}}{m_{3}^{2}}}
$$

for $\widetilde{R} / R=+1$, and

$$
\widetilde{x}=-\frac{\frac{m_{2}-m_{1}}{m_{2}+m_{1}}\left(1+\frac{m_{2}}{m_{3}}\right)+\frac{m_{2}}{m_{3}^{2}}\left(1-\frac{m_{2}}{m_{3}}\right)}{1+\frac{\left(m_{2}-m_{1}\right) m_{2}}{\left(m_{2}+m_{1}\right) m_{3}}+\frac{\left(m_{2}-m_{1}\right)\left(2 m_{2}+m_{1}\right) m_{1}}{\left(m_{2}+m_{1}\right) m_{3}^{2}}-\frac{m_{2} m_{1}^{2}}{m_{3}^{3}}}
$$

for $\widetilde{R} / R=+1$. The value of $\widetilde{x}$ is highly sensitive to the explicit value of $x$, so that we illustrate the behavior of $\widetilde{x}$ versus $x$ in Fig.1. Note that since $m_{1}^{2}=(1+R) m_{2}^{2}-R m_{3}^{3}$ from the definition (9), we obtain a constraint

$$
x^{2}>\frac{R}{1+R}
$$

for $R>0(\mathrm{NH})$, e.g. $|x|>0.165$ for the observed value $R=0.028$ [4, 5]. (For a case $R<0$ $(\mathrm{IH})$, the relation (14) is always satisfied, so that such a constraint does not appear.) In Fig.1 (a), (b) and (c), we have shown only cases in which the original model $M_{\nu}$ gives $\mathrm{NH}\left(x^{2}<1\right.$ and $R>0)$ and the inverse matrix model $\widetilde{M}_{\nu}$ gives NH $\left(\widetilde{x}^{2}<1\right.$ and $\left.\widetilde{R} / R=+1\right)$ or IH $\left(\widetilde{x}^{2}>1\right.$ and $\widetilde{R} / R=-1$ ) under the constraints $\Delta m_{21}^{2}>0$ and $\Delta \widetilde{m}_{21}^{2}>0$. As seen in Fig.1 (b), we find that we can always choose the value of $\widetilde{x}=\widetilde{m}_{2} / \widetilde{m}_{3}$ which can give IH with the same value $|\widetilde{R}|$ as $R$ by adding a suitable shift-term $m_{0} \xi_{0} \mathbf{1}$ to the inverse matrix $m_{0}^{2}\left(M_{\nu}^{*}\right)^{-1}$. Since the case (c) in Fig. 1 gives $1 / \widetilde{x}>0.3(1 / \widetilde{x} \sim 0.3$ at $x \sim 0.2)$, the case may be called as a case of a nearly degenerated hierarchy $(\mathrm{DH})$ rather than a case of $\mathrm{IH}$.

In this paper, we have interested in a case of $\mathrm{NH} \rightarrow \mathrm{IH}$. An inverse case, $\mathrm{IH} \rightarrow \mathrm{NH}$, can be guessed from the case of $\mathrm{NH} \rightarrow \mathrm{IH}$ [Fig.1 (b) and (c)]. For reference, we illustrate the remaining case, $\mathrm{IH} \rightarrow \mathrm{IH}$, in Fig.1 (d). As seen in Fig.1, the cases $\mathrm{NH} \rightarrow \mathrm{IH}$ and $\mathrm{IH} \rightarrow \mathrm{NH}$ take place in the case with $m_{1} / m_{2}<0$, and the cases $\mathrm{NH} \rightarrow \mathrm{NH}$ and $\mathrm{IH} \rightarrow \mathrm{IH}$ take place in the case with $m_{1} / m_{2}>0$.

So far, we have investigated a model under the constraints $T^{T} T=\mathbf{1}$ and $U_{\nu}^{T} U_{\nu}=\mathbf{1}$. However, the existence of such constraints narrows applicable cases of our statement to models. Finally, we would like to comment on a model with $\xi_{0}=0$. Then, a mass matrix $M_{\nu}$ can always 
provide the inverse matrix model $\widetilde{M}_{\nu} \equiv m_{0}^{2}\left(M_{\nu}^{*}\right)^{-1}$ which satisfies the same flavor symmetry $T^{T} \widetilde{M}_{\nu} T=\widetilde{M}_{\nu}$ and which has the same mixing matrix $U_{\nu}$ independently of whether $T^{T} T \neq \mathbf{1}$ and $U_{\nu}^{T} U_{\nu} \neq 1$ or not. The problem is whether such an inverse matrix $\widetilde{M}_{\nu}$ can also give a reasonable mass ratio $\widetilde{R}$ or not. As seen in Eq.(10), such a case gives

$$
\widetilde{R}=\left(\frac{m_{3}}{m_{1}}\right)^{2} R
$$

so that it seems to be ruled out because of the factor $\left(m_{3} / m_{1}\right)^{2}$ in Eq.(15). It is true as far as we want a model with an exact relation $|\widetilde{R}|=|R|$. However, the value of $\Delta m_{21}^{2} \equiv m_{2}^{2}-m_{1}^{2}$ is highly sensitive to the renormalization group equation (RGE) effects and some unknown loop corrections in a beyond-standard model. [The magnitudes of the effects are dependent on the model (the so-called $\tan \beta$ in SUSY model, and so on), so that the effects are not always conclusive.] For example, for a model of $M_{\nu}$ in which a predicted value of $\Delta m_{21}^{2}$ at a higher energy scale is too small value compared with the observed value of $\Delta m_{\text {solar }}^{2}$ and a predicted value of $\left(m_{3} / m_{1}\right)^{2}$ is not so large, it can be possible that the inverse matrix model $\widetilde{M}_{\nu}$ gives a reasonable value of $\widetilde{R}$. Therefore, we may expect that some of models $M_{\nu}$ can provide a reasonable masses and mixing for the inverse matrix model $\widetilde{M}_{\nu}$, too.

In conclusion, we have investigated whether a scenario which provides a matrix model $M_{\nu}$ with normal mass hierarchy can also give a model with an inverted mass hierarchy by considering a model with an inverse form of $M_{\nu}$. If the model $M_{\nu}$ is derived under a flavor symmetry $T^{T} M_{\nu} T=M_{\nu}$ with $T^{T} T=\mathbf{1}$, the inverse matrix model $\widetilde{M}_{\nu}$ which is defined by (2) can also satisfy the same symmetry as $T^{T} \widetilde{M}_{\nu} T=\widetilde{M}_{\nu}$, so that the scenario can give both model $M_{\nu}$ and $\widetilde{M}_{\nu}$. Then, we have find that if a model $M_{\nu}$ with normal hierarchy gives $m_{1} / m_{2}<0$, the inverse matrix model $\widetilde{M}_{\nu}$ can give a reasonable value of $\widetilde{R}$ with an inverted hierarchy. Even for a case without the constraints $T^{T} T=\mathbf{1}$ and $U_{\nu}^{T} U_{\nu}=\mathbf{1}$ (so that $\xi_{0}=0$ in Eq.(2)), we still have a possibility that the inverse matrix model $\widetilde{M}_{\nu}$ gives a reasonable value of $\widetilde{R}$ although we cannot give the exact relation $|\widetilde{R}|=|R|$. We may expect the observation of $\left\langle m_{e e}\right\rangle \sim 0.05 \mathrm{eV}$ in the near future neutrinoless double beta decay experiments by considering their inverse matrix models for a considerable number of current models.

\section{Acknowledgment}

This work is supported by the Grant-in-Aid for Scientific Research, Ministry of Education, Science and Culture, Japan (No.18540284).

\section{References}

[1] Y. Koide, Phys. Rev. D 71 (2005) 016010. For a review, Y. Koide, arXiv:0801.3491 [hepph], Invited talk presented at International Workshop on Grand Unified Theories: Current Status and Future Prospects, Ritsumeikan University, Kusatsu, Shiga, Japan, Dec. 17 - 19, 2007, to be published in the proceedings (AIP Conf. Proc.). 
[2] C. Froggatt and H. B. Nielsen, Nucl. Phys. B147 (1979) 277.

[3] T. Fukuyama and H. Nishiura, hep-ph/9702253, in Proceedings of the International Workshop on Masses and Mixings of Quarks and Leptons, Shizuoka, Japan, 1997, edited by Y. Koide (World Scientific, Singapore, 1998), p. 252; R. N. Mohapatra and S. Nussinov, Phys. Rev. D60, 013002, (1999); E. Ma and M. Raidal, Phys. Rev. Lett. 87, 011802 (2001); C. S. Lam, Phys. Lett. B507, 214 (2001); K. R. S. Balaji, W. Grimus and T. Schwetz, Phys. Lett. B508, 301 (2001); W. Grimus and L. Lavoura, Acta Phys. Pol. B32, 3719 (2001).

[4] D. G. Michael et al., MINOS collaboration, Phys. Rev. Lett. 97 (2006) 191801; J. Hosaka, et al., Super-Kamiokande collaboration, Phys. Rev. D74 (2006) 032002.

[5] S. Abe, et al., KamLAND collaboration, arXiv:0801.4589. 


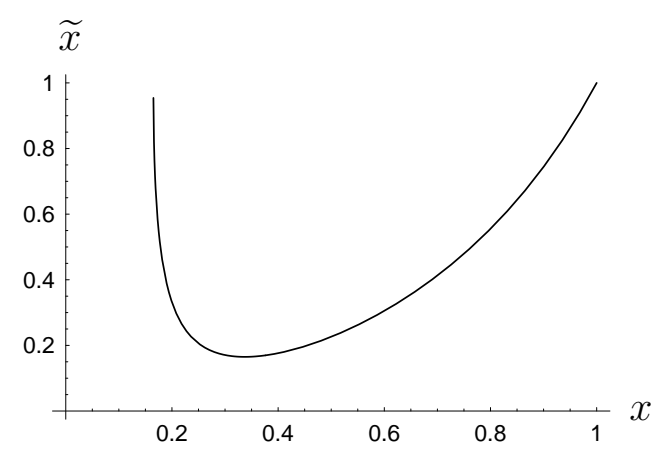

(a) Case $\mathrm{NH} \rightarrow \mathrm{NH}$

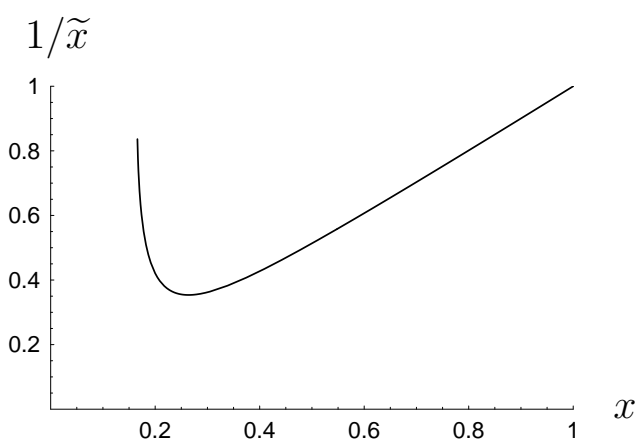

(c) Case $\mathrm{NH} \rightarrow \mathrm{IH}$

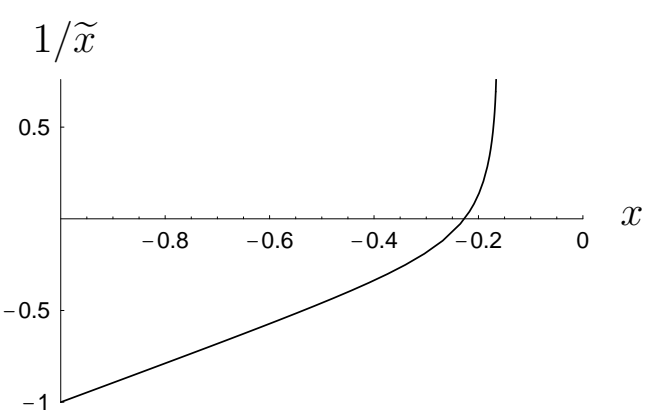

(b) Case $\mathrm{NH} \rightarrow \mathrm{IH}$

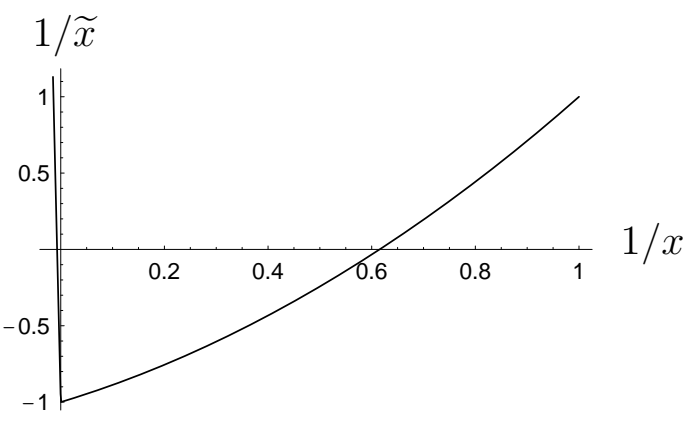

(d) Case $\mathrm{IH} \rightarrow \mathrm{IH}$

Fig. 1 Behaviors of $\widetilde{x}=\widetilde{m}_{2} / \widetilde{m}_{3}$ versus $x=m_{2} / m_{3}$ under the requirement of $|\widetilde{R}|=|R|$ : Input value $R=+0.028$ has been used as a NH case in $M_{\nu}$. (a) a case of $\mathrm{NH} \rightarrow \mathrm{NH}$ in $m_{1} / m_{3}>0$ and $m_{2} / m_{3}>0$; (b) a case of $\mathrm{NH} \rightarrow \mathrm{IH}$ in $m_{1} / m_{3}>0$ and $m_{2} / m_{3}<0$; (c) a case of $\mathrm{NH} \rightarrow \mathrm{IH}$ in $m_{1} / m_{3}<0$ and $m_{2} / m_{3}>0$; (d) a case of $\mathrm{IH} \rightarrow \mathrm{IH}$ in $m_{1} / m_{3}>0$ and $m_{2} / m_{3}>0$;. In the cases (b) and (c), NH $\rightarrow \mathrm{IH}$, the vertical axis is illustrated as $1 / \widetilde{x}$ instead of $\widetilde{x}$. Also, in the case $(\mathrm{d}), \mathrm{IH} \rightarrow \mathrm{IH}$, the horizontal and vertical axes are illustrated by $1 / x$ and $1 / \widetilde{x}$, respectively. 